\title{
The Effect of an In-Season 8-Week Plyometric Training Programme Followed By a Detraining Period on Explosive Skills in Competitive Junior Soccer Players
}

\author{
Luis Branquinho', Ricardo Ferraz ${ }^{1,2,3}$, Pedro Duarte Mendes ${ }^{4,5}$, João Petricia ${ }^{4,5}$, João Ser- \\ rano $^{4,5}$, Mário C. Marques 1,2
}

Affiliations: 'University of Beira Interior, Department of Sport Sciences, Covilhã, Portugal, ${ }^{2}$ Research Centre in Sports, Health and Human Development, Vila Real, Portugal, ${ }^{3}$ Football Association of Castelo Branco, Castelo Branco, Portugal 4Polytechnic Institute of Castelo Branco, Department of Sport and Well Being, Castelo Branco, Portugal, 5 Polytechnic Institute of Castelo Branco, Sport, Health \& Exercise Research Unit, Castelo Branco, Portugal

Correspondence: R. Ferraz, University of Beira Interior, Department of Sport Sciences, Convento de Santo António, 6201-001 Covilhã, Portugal. E-mail: ricardompferraz@gmail.com

ABSTRACT The aim of this study was to examine the effect of an eight-week plyometric training and resistance programme, on different motor abilities, kicking speeds, and individual techniques in competitive junior soccer players. A training programme was applied for eight weeks and was composed of countermovement jump, countermovement onto a box, sprints, and sprints with change of direction. The players were evaluated in three moments: before the start of the training programme, after the application of the training programme, and after four weeks of detraining. The applied tests were: countermovement jump, speed dribbling, 30-meter sprint, kicking speed, and a Yo-Yo recovery intermittent recovery test Level 2 (Yo-Yo IE2). The results showed statistically significant differences $(p<0.05)$ in sprint performance, countermovement jump, Yo-yo IE 2, and speed dribbling. The maximum velocity of the ball did not present statistically significant differences $(p>0.05)$. In addition, these results indicate that general performance may not be sensitive to four weeks of detraining in the trained soccer players. These results demonstrate that the combination of soccer drills and specific power training with no additional training time in-season optimizes some general and soccer-specific performance.

KEY WORDS explosive actions, soccer, young soccer players

$@ M J S S M o n t e n e g r o$

EFFECT OF AN IN-SEASON 8-WEEK PLYOMETRIC TRAINING PROGRAMME

http://mjssm.me/?sekcija=article\&artid=190

\section{Introduction}

During a soccer game, players perform a series of critical actions such as sprinting, changes of direction, jumps, ball recoveries, kicks and other actions which make a specific appeal to the ability to produce force quickly because such high levels of strength in the lower limbs are essential (Ramirez-Campillo et al., 2014). Based on the consensual importance of the explosive efforts in the outcome of a soccer game, strength training is crucial in order to make them physically prepared for competition (Rodriguez-Rosell et al., 2016). In this sense, explosive high-velocity training has demonstrated greater improvements in the rate of force development and explosive actions in comparison with traditional weight training methods for maximal strength (Wilson, Newton, Murphy, \& Humphries, 1993). This divergence could be explained by the absence of various crucial stimuli during strength training, in particular: segmental coordination, in regard to power transport by biarticular muscles, and neural control mechanisms for optimal movement patterns (Komi, 2008); specificity, according to joint angle and angular velocities (Komi, 2008); and eccentric overloading (de Hoyo et al., 2015).

Regarding this, plyometric training (PT) provides such training load stimuli and has been shown to improve

Received: September 032019 | Accepted after revision: October 162019 | First published online: March 012020

(c) 2020 by the author(s). License MSA, Podgorica, Montenegro. This article is an open access article distributed under the terms and conditions of the Creative Commons Attribution (CC BY).

Conflict of interest: None declared. 
significantly explosive actions in soccer players (Ramirez-Campillo et al., 2014). Additionally, PT is commonly used because, in addition to its easy application during training (space, time and equipment), it is also representative of neuromuscular demands found in soccer during explosive actions (i.e., sprinting and jumping) (Ferraz, van den Tillar, Pereira, \& Marques, 2016). Regarding this, a previous study demonstrated that high-intensity plyometric exercises could be used safely and effectively in soccer players to improve those abilities (Sáez de Villarreal, Requena, \& Cronin, 2012). Several other studies showed the benefits of a PT programme, specifically in young competitive soccer players (de Hoyo et al., 2015; Ramirez-Campillo et al., 2014). Moreover, significant relationships have been observed between lower limb strength and sprint time, vertical jumps, and changes of direction. A recent study by Chtara et al. (2017) presents PT as the most effective training programme to improve power performance among young soccer players. Nevertheless, Thomas, French, and Hayes (2009) observed, through the application of a six-week training programme, which included both squat (SJ) and countermovement jumps $(\mathrm{CMJ})$, improvements in vertical jump heights $(\mathrm{p}<0.05)$ in youth football players, although the performance remained the same in sprints $(\mathrm{p}<0.05)$. Another study experimented with PT for ten weeks, leading to distinctive results (Markovic, Jukic, Milanovic, \& Metikos, 2007), this study claimed improvements in SJs, height and power of the CMJs but no changes in sprint times.

Regarding the influence of PT in improving kicking velocity, some studies have shown that there is a positive impact on this ability (Wong, Chamari, \& Wisløf, 2010). However, the optimum training design for improving high-intensity actions is still not well established and needs to be found (Ramirez-Campillo et al., 2014). Some studies were unable to find any sign of improvements in this regard (Kraemer et al., 2002; Wong, Chamari, \& Wisløff, 2010), while others found some benefits after the application of PT (Marques \& González-Badillo, 2006).

Another topic that continues to require further clarification is related to the discontinuation of training sessions, an issue which may be due to various factors such as illnesses, injuries, vacations, post-season breaks or other normal factors in any type of sport, thus resulting in a reduction or cessation of their normal physical activity level (Kraemer et al., 2002; Marques \& González-Badillo, 2006). This phase of reduction or complete training cessation has been defined as "detraining" (Mujika \& Padilla, 2000). Several studies have reported that the magnitude of this reduction may depend upon the length of the detraining period (Kraemer et al., 2002), in addition to training levels attained by the subject (Izquierdo et al., 2007). Data from various athletic populations indicate that three to six weeks of detraining negatively affects aerobic capacity, strength (Izquierdo et al., 2007), neuromuscular performance (Izquierdo et al., 2007), and body composition. In contrast, according to Diallo, Dore, Duche, and Van Praagh (2001), a detraining period of eight weeks after the implementation of PT did not cause a decrease in the performance in prepubertal soccer players, suggesting that PT is beneficial for the youth soccer population. However, the detraining period and its consequences have not been completely clarified in the literature, particularly with respect to young soccer players. To the best of our knowledge, modifications in jumping, sprinting, kicking performance, aerobic performance, technical ability and impact on detraining as a result of a simple strength training programme with low volume, in addition to normal soccer training, have not been investigated as part of a study involving competitive junior soccer players.

Therefore, the objective of this study was to examine the effect of an eight-week strength training programme, consisting of explosive strength exercises for the lower extremities (e.g., sprints and jumps), on different motor abilities, kicking speed and individual technique in competitive junior soccer players. It also aimed to verify whether there was a positive impact on the reduction of loss of physical capacity after a period of detraining, with respect to gains previously achieved. It was hypothesized that the training group would enhance their jumping, sprinting, and aerobic performance. Enhancement of technical ability and kicking performance were also hypothesized, due to an expected increase in explosive strength established by the PT programme.

Furthermore, it is expected that there will be a positive impact on the effects of detraining. Because of ethical considerations, it was not possible to include a control group, so all athletes completed the same in-season resistance-training programme. Experimental studies in competitive athletes, especially in team sports, are very difficult to put into practice (Marques \& González-Badillo, 2006; Marques, van den Tillaar, Vescovi, \& González-Badillo, 2008). These difficulties are compounded by a problem discussed by Marques et al. (2008). In practical terms, to locate a specific control group (i.e., another elite team volleyball sample with the same training and at the same performance level as the experimental team) and to access testing conditions is not an easy task for coaches or researchers. However, such considerations ought not to detract from the necessity and importance of this type of investigation or of the present case report, especially in volleyball.

\section{Methods}

Subjects

A group of 15 young male soccer players belonging to a Portuguese team playing at the national level in their age category under 18 years (Mean \pm SD age: $17.27 \pm 0.458$ years) participated in the study, with none of the participants having regular habits of strength training. With respect to the characteristic anthropometrics, the group presents the following data: weight $(68.8 \pm 9.77 \mathrm{~kg})$ height $(1.76 \pm 0.07 \mathrm{~m})$. Before the study started, players had a physical examination by the team physician, and each was cleared of any medical disorders that may have limited full participation in the investigation. All participants and the coach were fully informed verbally and in writing regarding the nature and demands of the study, as well as the known health risks. They completed a health history questionnaire and were informed that they could withdraw from the study at any time, even after giving their 
written consent. All parents provided their informed consent attesting the voluntary participation of their children in the study, which had the approval of the Academy's Ethical Advisory Commission, and was conducted in accordance with the Declaration of Helsinki.

\section{Experimental design}

The objective of this study was to examine the effect of an eight-week PT programme, consisting of explosive strength exercises for the lower extremities, on different motor abilities, kicking speed and individual technique in competitive junior soccer players. The training programme took place during a period of eight weeks followed by four weeks of detraining. In addition, to allow us to realize the effects of PT as a complement to specific technical and tactical training, the detraining period enabled us to better understand the impact of this complementary training. All players competed in one match per week combined with four soccer practice sessions. Players had completed a pre-season testing and training programme prior to the initiation of this in-season study. The players were in good physical condition and were adequately familiarized with all procedures prior to commencing the study. Apart from standard technical and tactical practice sessions (two hours per day) and regular competitions, the subjects completed a physical training regimen that included lower-body exercises targeting strength and power. All experimental procedures were carried out in coordination with the technical team and so did not cause any change in the routine of the players. All testing was carried out during one week at the completion of the second half of the in-season, which took place between January and April. Before the pre-test stage, the participants were familiarized with the different tests during a practice session in order to minimize learning effects. Pre- and post-tests were performed with maximal intensity. All tests were conducted in an indoor facility in order to eliminate the effect of weather conditions on results. The players were evaluated at three different times: before the start of the training programme (Pre-training test) (T1), after the application of the training programme (Post-training test) (T2) and after four weeks of detraining (Post - detraining test) (T3).The tests conducted during the current study were: countermovement jump, speed dribbling, 30-metre sprint, kicking speed and a Yo-Yo recovery intermittent recovery test Level 2 (Yo-Yo IE2). Tests were performed over a 3-day period: Say 1: anthropometric measures, countermovement jump, speed dribbling; Day 2: 30-metre sprint and Speed Shooting; Day 3: (Yo-Yo IE2). These were tests that could be rapidly administered and were highly specific to soccer.

\section{Procedures}

The soccer history of each player and their game positions were determined using a questionnaire. The anthropometric variables of height and body mass were measured for each subject, on a levelled platform scale (Año Sayol, Barcelona, Spain) with an accuracy of $0.001 \mathrm{~m}$ and $0.01 \mathrm{~kg}$, respectively.

\section{Warm up}

Before each of the exercises, a warm-up was performed based on the protocol described by van den Tillaar, Lerberg, and von Heimburg (in press). The short specific warm-up consisted of $8 \times 60 \mathrm{~m}$ runs with $60 \mathrm{~s}$ rest in between $(10 \mathrm{~min}$ in total). The first $60 \mathrm{~m}$ was performed at a self-estimated intensity of around $60 \%$ of estimated maximal sprinting velocity. Every next $60 \mathrm{~m}$ was increased by around 5\% until it reached $95 \%$ of maximal self-estimated intensity. In each rest period, one of the same seven dynamic exercises as in the other two warm-up protocols was used.

\section{$C M J$}

CMJ height was measured using a trigonometric carpet (Ergo jump Digitimer 1000, Digest Finland) using previously described methods (Marques, van den Tillaar, Vescovi, \& González-Badillo, 2008). Subjects began from a standing position, performed a crouching action followed immediately by a jump for maximal height. The hands were on the hips during the entire jump. Each participant performed three jumps, and the highest jump was recorded. Between each repetition, there was a two-minute rest period.

\section{Sprint}

The 30-m sprint was performed in an indoor school physical education facility with a Copolymer Polypropylene floor, with subjects wearing indoor shoes. The time required to run $30 \mathrm{~m}$ was obtained using photocells (Brower Timing System, Fairlee, Vermont, USA). Times at 10, 20 and $30 \mathrm{~m}$ were also recorded. Prior to each sprint, each subject trod the cell pad using the right hand, with the time being recorded from when the subject intercepted the photocell beam. All subjects were encouraged to run as fast as possible and to decelerate only after listening to the beep emitted by the last pair of photocells. Each player repeated the same procedure for three attempts, with only the best time taken to cover the $30-\mathrm{m}$ distance in the sprint test being used in data analysis. A rest period of $10 \mathrm{~min}$ was provided between attempts.

\section{Kicking Speed}

For the kicking speed and accuracy test, a standard soccer ball (mass approximately $430 \mathrm{~g}$, circumference 70 $\mathrm{cm}$ ) was used. After a general warm-up of $15 \mathrm{~min}$, which included jogging and kicking drills, kicking performance was tested. The instruction was to kick a regular ball with maximum force and attempt to hit a target from an $11-\mathrm{m}$ distance, aiming at a $1 \mathrm{~m}$ by $1 \mathrm{~m}$ circled target at $2 \mathrm{~m}$ height located in the middle of a goal (3 $\times 2 \mathrm{~m}$ ). The kicking velocity of the ball was determined using a Doppler radar gun (Sports Radar 3300, Sports 
Electronics Inc.), with $\pm 0.028 \mathrm{~m} \cdot \mathrm{s}-1$ accuracy within a field of ten degrees from the gun. The radar gun was located $1 \mathrm{~m}$ behind the goal at ball height. Three trials were conducted, and the highest ball kicking velocity was used for further analysis.

\section{Dribbling Speed}

Dribbling with the ball is a skill required by all players but is particularly effective for those attacking players looking to break open a defence by running at opponents through tight spaces. This drill tests a player's ability to dribble the ball rapidly through a marked circuit, where good performance relies on close ball control and the ability to maintain control during rapid changes of direction. Dribbling speed was quantified by recording the total time taken for an individual to dribble (i.e., kick) the soccer ball through a 61.2-m agility course. Each individual was given three attempts at the task, with a 2.5 -min rest between each and the quickest being taken as their peak performance (for all skill tests, an individual's peak performance was their single best performance in that task). Each player started with the ball behind the first cone and proceeded through the circuit as fast as possible. Time was stopped when both the player and ball crossed the finish line. The time taken to complete the circuit was recorded with a stopwatch and then converted to an average speed over the $61.2 \mathrm{~m}$. Time penalties were allotted to a player's total time to account for errors using the following system: (i) $+1 \mathrm{~s}$ for each missed cone, (ii) $+2 \mathrm{~s}$ if two cones in succession were missed (note: although this penalty was applied, it was never part of an individual's quickest time, which was taken as their peak performance), and (iii) $+0.5 \mathrm{~s}$ for each cone knocked over. Repeatability was calculated by comparing a player's first and second tests of dribbling speed.

\section{The Yo-yo recovery intermittent test Iv 2}

All players $(\mathrm{n}=15)$ completed the Yo-Yo IE2 test at the beginning and at the end of the training programme. The test lasts 5-25 min and consists of repeated 20-m shuttle runs at progressively increasing speeds dictated by an audio beep emitted from a CD player. Between each shuttle, the players had a 10-s period of jogging around a marker placed $5 \mathrm{~m}$ behind the finishing line. Failure to achieve the shuttle run in time on two occasions resulted in termination of the test and the distance covered in the last complete successful shuttle was recorded as the test result. All testing sessions were performed indoors. Before the test, all players carried out a warm-up period consisting of the first three running bouts of the Yo-Yo IE2 test followed by a period of lower-extremity stretching. All players had previously been familiarized with the Yo-Yo IE2 test and experimental procedures.

\section{Training Programme}

Briefly, the programme was performed twice per week, with each session lasting approximately 20 minutes and performed just after the warm-up to ensure that the players were in a rested state and gained optimal

\begin{tabular}{|c|c|c|c|c|c|}
\hline & & CMJ & CMJ OB & Sprint & SCOD \\
\hline \multirow{2}{*}{ Week 1} & Session 1 & $2 \times 4$ & $2 \times 4$ & $2 \times 20 m$ & $2 \times 10 s$ \\
\hline & Session 2 & $2 \times 4$ & $2 \times 4$ & $3 \times 20 m$ & $2 \times 10 s$ \\
\hline \multirow{2}{*}{ Week 2} & Session 3 & $2 \times 4$ & $3 \times 4$ & $2 \times 20 m$ & $3 \times 10 s$ \\
\hline & Session 4 & $3 \times 4$ & $2 \times 4$ & $3 \times 20 \mathrm{~m}$ & $2 \times 10 s$ \\
\hline \multirow{2}{*}{ Week 3} & Session 5 & $3 \times 4$ & $3 \times 4$ & $3 \times 20 m$ & $3 \times 10 s$ \\
\hline & Session 6 & $3 \times 4$ & $2 \times 4$ & $3 \times 20 m$ & $3 \times 10 s$ \\
\hline \multirow{2}{*}{ Week 4} & Session 7 & $3 \times 4$ & $3 \times 4$ & $3 \times 20 m$ & $4 \times 10 s$ \\
\hline & Session 8 & $3 \times 5$ & $2 \times 4$ & $4 \times 20 m$ & $3 \times 10 s$ \\
\hline \multirow{2}{*}{ Week 5} & Session 9 & $3 \times 4$ & $3 \times 5$ & $3 \times 20 m$ & $4 \times 10 s$ \\
\hline & Session 10 & $3 \times 5$ & $2 \times 5$ & $4 \times 20 m$ & $3 \times 10 s$ \\
\hline \multirow{2}{*}{ Week 6} & Session 11 & $3 \times 4$ & $3 \times 5$ & $2 \times 30 \mathrm{~m}$ & $4 \times 10 s$ \\
\hline & Session 12 & $4 \times 4$ & $3 \times 5$ & $3 \times 20 m$ & $3 \times 10 s$ \\
\hline \multirow{2}{*}{ Week 7} & Session 13 & $4 \times 4$ & $3 \times 5$ & $3 \times 30 m$ & $4 \times 10 s$ \\
\hline & Session 14 & $4 \times 5$ & $3 \times 4$ & $3 \times 20 \mathrm{~m}$ & $3 \times 10 s$ \\
\hline \multirow{2}{*}{ Week 8} & Session 15 & $3 \times 5$ & $3 \times 4$ & $2 \times 20 m$ & $3 \times 10 s$ \\
\hline & Session 16 & $3 \times 4$ & $2 \times 4$ & $2 \times 20 m$ & $1 \times 10 \mathrm{~s}$ \\
\hline
\end{tabular}

Note. CMJ - counter-movement jump, CMJ OB- counter-movement jump onto a box, SPRINT - 20-metres sprint, SCOD - Sprint with change of direction. 
benefits from the specific programme, according to the training principle of priority. The training programme was applied for eight weeks and was composed of countermovement jump (CMJ), countermovement onto a box, sprints and sprints with change of direction as shown in Table 1.

\section{Statistical analysis}

The normality of all distributions was verified by the Shapiro-Wilk test, and the t-test analysis was used to compare means from the sets of data. Standard statistical procedures were selected for the calculation of means, standard deviations and 95\% confidence intervals. The level of significance was set at $\mathrm{p}<0.05$. The statistical analysis was performed with SPSS version 22.0 (SPSS, Inc., Chicago, IL, USA). The effect size was evaluated with $\mathrm{d}$ (Cohen's $\mathrm{d}$ ), where $0.20<\mathrm{d}<0.30$ constitutes a small effect, $0.40<\mathrm{d}<0.70$ constitutes a medium effect, and $\mathrm{d} \geq 0.80$ indicates a large effect.

\section{Results}

The three moments of evaluation were paired, as shown below for analysis: T1 / T2; T2 / T3; T1 / T3. In sprint performance, statistically significant differences $(\mathrm{p}<0.05)$ were found between T1 / T2; T2 / T3; T1 / T3, with an effect size $(d=0.51) ;(D=0.41) ;(D=0.04)$. The CMJ performance changed significantly during the training application, $\mathrm{T} 1 / \mathrm{T} 2(\mathrm{p}<0.05 ; \mathrm{d}=0.51)$. However, the maximum velocity of the ball did not present statistically significant differences between the three moments analysed $(\mathrm{p}>0.05, \mathrm{~d}=0.44, \mathrm{~d}=0.10, \mathrm{~d}=0.35$ ). The variables analysed in the Yo-yo IE 2 obtained different results. For stage distance, statistically significant differences were found between T1 / T2 $(\mathrm{p}<0.05)$. For the distance travelled, statistically significant differences in T1 / T2 ( $>0.05)$ were not observed; however, the T2 / T3; T1 / T3 showed statistically significant differences, despite there only being a small effect size $(d=0.02 ; d=0.17)$. The initial lactate concentration did not change significantly before starting the Yo-Yo IE2 ( $p>0.05$ ), contrary to what happened with the final concentration of lactate, with which there were statistically significant differences $(\mathrm{p}<0.05)$ with a high effect size $(\mathrm{d}=0.71 ; \mathrm{d}=0.55 ; \mathrm{d}=1.21)$, respectively. For speed dribbling, the best time achieved at each of the evaluated moments was analysed. In the analysis of the T1 / T2, no differences were found ( $>>0.05)$, but the same did not occur for T2 / T3; T1 / T3 where there were statistically significant differences $(\mathrm{p}<0.05 ; \mathrm{d}=0.30 ; \mathrm{d}=$ 0.35 ) as shown in Tables 2 .

\begin{tabular}{|c|c|c|c|c|c|c|c|c|c|c|c|c|c|}
\hline & & \multirow{2}{*}{$\begin{array}{c}\begin{array}{c}\text { Pre- } \\
\text { training }\end{array} \\
\text { (T1) }\end{array}$} & \multirow{2}{*}{$\begin{array}{c}\begin{array}{c}\text { Post- } \\
\text { training }\end{array} \\
\text { (T2) }\end{array}$} & \multirow{2}{*}{$\begin{array}{c}\text { detraining } \\
\text { (T3) }\end{array}$} & \multicolumn{3}{|c|}{ T1 vs. T2 } & \multicolumn{3}{|c|}{ T2 vs. T3 } & \multicolumn{3}{|c|}{ T1 vs. T3 } \\
\hline & & & & & $\mathbf{p}$ & $\Delta(\%)$ & d & $\mathbf{p}$ & $\Delta(\%)$ & D & $\mathbf{p}$ & $\Delta(\%)$ & d \\
\hline CMJ & & $32.33 \pm 5.25$ & $35.06 \pm 5.39$ & $34.16 \pm 5.16$ & $0.001^{* * *}$ & 8.44 & 0.51 & $0.001^{* * *}$ & -2.57 & 0.17 & $0.005^{* *}$ & 5.66 & 0.35 \\
\hline Sprint 30m & & $4.54 \pm 0.015$ & $4.46 \pm 0.14$ & $4.55 \pm 0.19$ & $0.015^{*}$ & -1.76 & 0.51 & 0.015 & 2.02 & 0.49 & 0.834 & 0.22 & 0.04 \\
\hline $\begin{array}{l}\text { Kicking } \\
\text { speed }\end{array}$ & & $90.66 \pm 3.58$ & $92.11 \pm 2.89$ & $91.82 \pm 2.80$ & 0.067 & $1 . .60$ & 0.44 & $0.489^{*}$ & -0.31 & 0.10 & 0.069 & 1.28 & 0.35 \\
\hline $\begin{array}{l}\text { Dribbling } \\
\text { speed }\end{array}$ & & $25.04 \pm 1.79$ & $24.93 \pm 1.64$ & $24.45 \pm 1.48$ & 0.730 & -0.44 & 0.06 & $0.013^{*}$ & -1.93 & 0.30 & $0.023^{*}$ & -2.36 & 0.35 \\
\hline \multirow{4}{*}{ Yo-Yo IE } & Stage & $11.60 \pm 3.26$ & $12.20 \pm 3.05$ & $12.13 \pm 2.47$ & $0.007^{* *}$ & 5.17 & 0.18 & 0.751 & -0.57 & 0.02 & 0.088 & 4.57 & 0.17 \\
\hline & Distance & $464 \pm 130.7$ & $488 \pm 128.4$ & $488 \pm 101.6$ & $0.014^{*}$ & 5.17 & 0.18 & 1.000 & 0 & 0 & 0.057 & 5.17 & 0.20 \\
\hline & $\begin{array}{l}\text { Blood lactate } \\
\text { BT }\end{array}$ & $1.51 \pm 0.28$ & $1.60 \pm 0.28$ & $1.66 \pm 0.21$ & 0.657 & -5.96 & 0.32 & 0.454 & 3.75 & 0.23 & 0.163 & 9.93 & 0.59 \\
\hline & $\begin{array}{l}\text { Blood lactate } \\
\text { AT }\end{array}$ & $10.10 \pm 1.42$ & $9.16 \pm 1.17$ & $8.52 \pm 1.13$ & $0.008^{* *}$ & -9.31 & 0.71 & $0.001 * * *$ & -6.99 & 0.55 & $0.001^{* * *}$ & 15.64 & 1.21 \\
\hline
\end{tabular}

Note. $\mathrm{p}$ - $\mathrm{p}$-value, $\Delta(\%)$ - Pre - post change, $\mathrm{d}$ - Cohens $\mathrm{d}$. ${ }^{*}$ indicates $\mathrm{P}<0.05,{ }^{* *}$ indicates $\mathrm{P}<0.01$ and ${ }^{* * *}$ indicates $\mathrm{P}<0.001$.

\section{Discussion}

The main aim of this study was to examine the effect of an eight-week PT training programme on different motor abilities and also on VO2 max in competitive junior soccer players. In addition, it was intended to verify the influence of four weeks of detraining on the minimization of losses in relation to the previously achieved gains. It was found that the training programme significantly improved sprint ability and CMJ. However, kicking speed, speed dribbling and Yo-yo IE 2 did not show significant improvements in the T2 period. Therefore, these results demonstrate that the combination of soccer drills and specific explosive training with no additional training time in-season optimize some general and soccer-specific explosiveness and endurance performance in junior soccer payers. In addition, the results of this investigation indicate that short-term PT 
using moderate training frequency and low volume produces similar enhancements in sprint time and CMJ compared with high training and volume, which is in accordance with what was previously been reported (de Villarreal, Requena and Cronin, 2012).

As a result of the application of a PT programme of low volume and high intensity, there was a decrease in the time of the 30-m sprint. These results are contrary to other studies showing no improvements in sprint time after a plyometric programme (Fry et al., 1991; Wilson, Newton, Murphy and Humphries, 1993). However, recent investigations including a meta-analysis (Sáez de Villarreal et al., 2012) have also found a decrease in sprint times with PT (Sáez de Villarreal et al., 2012). Earlier studies have reported no significant increases in sprint acceleration or velocity after training programmes involving essentially vertical plyometric exercises and weight training in trained subjects (Fry et al., 1991; Wilson et al., 1993). As the training stimulus applied during the study was vertical and horizontal in nature, this set-up could have increased the chances of soccer players becoming adapted, considering the importance of horizontal force production and the application in running performance (Morin et al., 2012) and the principle of specificity of training (Sáez de Villarreal et al., 2012). Gains of sprint performance reflect neural adaptations such as an increased nerve conduction velocity, maximizing of the electromyogram, improved intermuscular coordination, an enhanced motor unit recruitment strategy, and an increased excitability of the Hoffman reflex (H-reflex) (Markovic \& Mikulic, 2010) as well as changes in muscle size and architecture, in the mechanical characteristics of the muscle-tendon complex and changes in single-fibre mechanics (Markovic \& Mikulic, 2010). Thus, it is apparent that the PT performed during the regular soccer practices in the current study may be important for sprint adaptations. To the best of our knowledge, this is one of the few studies to investigate the effect of a PT with low volume and high intensity on the sprint performance of junior soccer players.

The plyometric programme was also effective in significantly increasing jump height of CMJ. The improvement in CMJ height indicates that adaptations relating to increases in leg power occurred. Improvements of muscle power and vertical jump height with PT have been described previously (Markovic \& Mikulic, 2010), and our results are consistent with these findings. The adaptation occurred in response to the type of training that was applied is probably neural because it predominates in the initial stages of strength and power training, and has been shown to be the main adaptation to plyometric exercise. In addition, this phenomenon may be because the $\mathrm{CMJ}$ involves a stretch-shortening cycle and is thus very similar to the one plyometric exercise used in our study. Moreover, PT is likely to improve coordination and thus induces a neuromuscular adaptation that augments power production (Sáez de Villarreal et al., 2012). The properly developed ability to jump can be a determining factor in the success during a game, given its importance in the dispute of the ball in offensive and defensive actions during a game.

Kicking performance did not change significantly after PT application. Although several studies have reported improvements in kicking ball velocity performance as a result of specific strength training (Ferraz et al., 2016; Wong et al., 2010), this improvement was not observed in our study. However, the data found are in agreement with that described in an earlier study, in which the data revealed that although six weeks of PT was sufficient to produce significant improvements in explosive strength, players required 12 weeks to produce significant increases in kicking speed. The improvements in kicking speed after PT could be attributed to a change in some kinematic variables, such as linear velocity of the distal segments and the position of the body throughout the shot. Possibly, these changes promote an adaptation in the kicking movement after gains in strength and may be the result of an altered SCC of musculature involved (Ferraz, van den Tillar, \& Marques, 2017; Wong et al., 2010). This could be the reason for the lack of significant improvements in kicking speed after eight weeks of PT.

The results showed that the time of dribbling speed did not improve significantly after PT application. Speed dribbling mainly requires agility in its execution, a factor that is defined as the ability to change the direction of the body abruptly; thus, there seems to be a positive relationship between agility and dribbling performance, with an earlier study speculating that improvements in agility were a result of enhanced motor unit recruitment patters (Potteiger et al., 1999). Neural adaptations occur mainly when athletes respond or react as a result of improved coordination between the central nervous system signal and proprioceptive feedback. These adaptations occurred via synchronous firing of the motor neurons or better facilitation of neural impulses to the spinal cord, further supporting the suggestions of a previous study (Potteiger et al., 1999). However, we cannot determine the reason for the lack of improvements. In addition, the ability to sprint is another of the skills required to complete the circuit quickly. Although sprint capacity has been optimized (30 metres) with PT application, the total distance of the circuit is higher, which may help to justify the lack of significant improvements. Furthermore, it has been reported that strength training does not always improve $20-100 \mathrm{~m}$ sprint times (Kotzamanidis, Chatzopoulos, Michailidis, Papaiakovou, \& Patikas, 2005).

To the best of our knowledge, this is the first study to demonstrate the effects of a low volume PT in the Yo-Yo IR2 performance in a group of young competitive soccer players. Our results demonstrated that the stage variable improved significantly after PT application. These results are in line with those described in previous studies (Barnes \& Kilding, 2015). One of the possible explanations may be related to a better running economy, which in turn might be explained by decreased ground contact times increasing musculotendinous stiffness, elastic energy returns neuromuscular activity or enhanced running mechanics. The final blood lactate values change significantly during the three analysed moments. The increment of blood lactate tolerance at submaximal intensity seems to be a key factor in avoiding diminished performance on repeated high-intensity 
exercise, such as the Yo-Yo IR2 (Nakamura, Suzuki, Yasumatsu, \& Akimoto, 2012). These data can help to justify the maintenance of the values of some variables (stage, distance) during the four weeks of detraining. In addition, a weak but significant relation between Yo-Yo IR2 performance and VO2 max has been shown in samples consisting of both professional and amateur players (Rampinini et al., 2010), which is in agreement with our findings, where it is described that for the VO2 max, there were few significant alterations after PT application. Soccer players may suffer interruptions in the training process and competitive calendars, which may result in a reduction of the normal parameters of physical capacity (Kraemer et al., 2002). With regard to the four-week DT, our findings revealed that the detraining period failed to bring out significant differences from amongst all the parameters, with the exception of sprint performance. These results are similar to others previously reported (Nakamura et al., 2012), indicating that short duration and high-intensity exercise performances did not change after short-term training cessation ( $<4$ weeks). Previous studies have also reported that the detraining period in which the regular training of a specific sport is maintained allows an athlete to maintain the gains achieved (Marques \& González-Badillo, 2006). However, these results are different from others reported previously (Hakkinen, Alen, \& Komi, 1985), where significant reductions were specifically observed in the CMJ after 12 weeks of DT; these identified differences may be due to a longer detraining period (Marques \& González-Badillo, 2006) The inability to stimulate motor units or fast contraction fibres required for explosive abilities raises the hypothesis that the absence of training stimulus causes significant neural losses to the muscles. However, with a shorter detraining period between 2-7 weeks the jump performance seems could be maintained (Kraemer et al., 2002). In our study, the reduced impact of detraining period can be a good indicator of the positive impact resulting from the PT used to increase the performance of junior soccer players

In addition, these results indicate that anaerobic exercise performance may not be sensitive to short-term detraining in the trained soccer players. Several possibilities may explain these findings in the trained athletes. It may be that the reason for decreased sprint performance is related to accumulated fatigue through the course of the soccer season. Another possible mechanism for increased sprint performance after detraining is likely to be related to the adaptation in anaerobic enzyme activities in human skeletal muscle (Joo, 2016). Furthermore, insufficient training stimulus or training cessation are commonly associated with reductions in blood volume, stroke volume, cardiac output, ventilator functions and cardiac dimension, all of which may have been associated with the differences found (Mujika \& Padilla, 2000). Future studies are, therefore, required. In summary, the data from the present study support the assumption that a PT programme with low volume and high intensity may be a suitable training method for obtaining strength improvements.

In conclusion, the current study demonstrates that the combination of soccer drills and specific power training with no additional training time in-season optimize some aspects of general and soccer-specific explosiveness and endurance performance in junior soccer payers. Also showed that four weeks of detraining period are not sufficient to cause significant losses in the explosive variables required to soccer players. The data support the application of PT programmes during the season. This type of programme is easy to apply and occupied a short time in the training unit. In addition, also has the added advantage that it can be applied without the need for large investments by the clubs, which makes it applicable to all clubs in the world.

\section{Acknowledgements}

This work was founding by "Bolsa BID/ICI-FCSH/Santader Universidades-UBI 2016. This work is supported by national funding through the Portuguese Foundation for Science and Technology, I.P., under project UID / DTP / 04045/2019.

\section{References}

Barnes, K. R., \& Kilding, A. E. (2015). Strategies to improve running economy. Sports medicine 45(1), 37-56. doi: 10.1007/s40279-014-0246-y

Chtara, M., Rouissi, M., Haddad, M., Chtara, H., Chaalali, A., Owen, A., \& Chamari, K. (2017). Specific physical trainability in elite young soccer players: efficiency over 6 weeks' in-season training. Biology of sport, 34(2), 137-148. doi:10.5114/biolsport.2017.64587

de Hoyo, M., Pozzo, M., Sañudo, B., Carrasco, L., Gonzalo-Skok, O., Domínguez-Cobo, S., \& Morán-Camacho, E. (2015). Effects of a 10-Week In-Season Eccentric-Overload Training Program on Muscle-Injury Prevention and Performance in Junior Elite Soccer Players. International journal of sports physiology and performance, 10(1), 46-52. doi: 10.1123/ijspp.2013-0547

Diallo, O., Dore, E., Duche, P., \& Van Praagh, E. (2001). Effects of plyometric training followed by a reduced training programme on physical performance in prepubescent soccer players. The Journal of sports medicine and physical fitness, 41(3), 342-348.

Ferraz, R. M. P., van den Tillaar, R., Pereira, A., \& Marques, M. C. (in Press). The effect of fatigue and duration knowledge of exercise on kicking performance in soccer players. Journal of Sport and Health Science. doi: $10.1016 / j$.jshs.2016.02.001

Ferraz, R., van den Tillar, R., \& Marques, M. C. (2017). The influence of different exercise intensities on kicking accuracy and velocity in soccer players. Journal of Sport and Health Science, 6(4), 462-467. doi: 10.1016/j.jshs.2015.10.001

Fry, A. C., Kraemer, W. J., Weseman, C. A., Conroy, B. P., Gordon, S. E., Hoffman, J. R., \& Maresh, C. M. 
(1991). The Effects of an Off-season Strength and Conditioning Program on Starters and Non-starters in Women's Intercollegiate Volleyball. Journal of Strength \& Conditioning Research, 5(4), 174-181.

Hakkinen, K., Alen, M., \& Komi, P. V. (1985). Changes in isometric force- and relaxation-time, electromyographic and muscle fibre characteristics of human skeletal muscle during strength training and detraining. Acta Physiologica Scandinavica. Supplementum, 125(4), 573-585. doi: 10.1111/j.1748-1716.1985.tb07760.x

Izquierdo, M., Ibanez, J., Gonzalez-Badillo, J. J., Ratamess, N. A., Kraemer, W. J., Hakkinen, K., ... Gorostiaga, E. M. (2007). Detraining and tapering effects on hormonal responses and strength performance. Journal of strength and conditioning research, 21(3), 768-775. doi: 10.1519/r-21136.1

Joo, C. H. (2016). The effects of short-term detraining on exercise performance in soccer players. Journal of Exercise Rehabilitation, 12(1), 54-59. doi: 10.12965/jer.160280

Komi, P. (2008). Strength and power in sport. Hoboken, NJ: John Wiley \& Sons.

Kotzamanidis, C., Chatzopoulos, D., Michailidis, C., Papaiakovou, G., \& Patikas, D. (2005). The effect of a combined high-intensity strength and speed training program on the running and jumping ability of soccer players. Journal of Strength \& Conditioning Research, 19(2), 369-375.

Kraemer, W. J., Koziris, L. P., Ratamess, N. A., HÄkkinen, K., Triplett-mcbride, N. T., Fry, A. C., .. . Rubin, M. R. (2002). Detraining produces minimal changes in physical performance and hormonal variables in recreationally strength-trained men. Journal of Strength \& Conditioning Research, 16(3), 373-382.

Markovic, G., \& Mikulic, P. (2010). Neuro-musculoskeletal and performance adaptations to lower-extremity plyometric training. Sports Medicine, 40(10), 859-895. doi: 10.2165/11318370-000000000-00000

Markovic, G., Jukic, I., Milanovic, D., \& Metikos, D. (2007). Effects of sprint and plyometric training on muscle function and athletic performance. Journal of Strength \& Conditioning Research, 21(2), 543-549. doi: 10.1519/R-19535.1

Marques, M. C., van den Tillaar, R., Vescovi, J. D., \& González-Badillo, J. J. (2008). Changes in strength and power performance in elite senior female professional volleyball players during the in-season: a case study. Journal of Strength \& Conditioning Research, 22(4), 1147-1155. doi: 10.1519/JSC.0b013e31816a42d0

Marques, M., \& González-Badillo, J. (2006). In-season resistance training and detraining in professional team handball players. Journal of strength and conditioning research, 20(3), 563-571.

Morin, J.-B., Bourdin, M., Edouard, P., Peyrot, N., Samozino, P., \& Lacour, J.-R. (2012). Mechanical determinants of 100-m sprint running performance. European Journal of Applied Physiology, 112(11), 3921-3930. doi: 10.1007/s00421-012-2379-8

Mujika, I., \& Padilla, S. (2000). Detraining: loss of training-induced physiological and performance adaptations. Part I. Sports Medicine, 30(2), 79-87.

Nakamura, D., Suzuki, T., Yasumatsu, M., \& Akimoto, T. (2012). Moderate running and plyometric training during off-season did not show a significant difference on soccer-related high-intensity performances compared with no-training controls. Journal of strength and conditioning research, 26(12), 3392-3397. doi: 10.1519/JSC.0b013e3182474356

Potteiger, J. A., Lockwood, R. H., Haub, M. D., Dolezal, B. A., Almuzaini, K. S., Schroeder, J. M., \& Zebas, C. J. (1999). Muscle power and fiber characteristics following 8 weeks of plyometric training. Journal of Strength and Conditioning Research, 13, 275-279.

Ramirez-Campillo, R., Meylan, C., Alvarez, C., Henriquez-Olguin, C., Martinez, C., Canas-Jamett, R., . . . Izquierdo, M. (2014). Effects of in-season low-volume high-intensity plyometric training on explosive actions and endurance of young soccer players. Journal of strength and conditioning research, 28(5), 13351342. doi: $10.1519 /$ jsc.0000000000000284

Rampinini, E., Sassi, A., Azzalin, A., Castagna, C., Menaspa, P., Carlomagno, D., \& Impellizzeri, F. M. (2010). Physiological determinants of Yo-Yo intermittent recovery tests in male soccer players. European journal of applied physiology, 108(2), 401-409. doi: 10.1007/s00421-009-1221-4

Rodriguez-Rosell, D., Franco-Marquez, F., Pareja-Blanco, F., Mora-Custodio, R., Yanez-Garcia, J. M., GonzalezSuarez, J. M., \& Gonzalez-Badillo, J. J. (2016). Effects of 6 Weeks Resistance Training Combined With Plyometric and Speed Exercises on Physical Performance of Pre-Peak-Height-Velocity Soccer Players. International Journal of Sports Physiology Performance, 11(2), 240-246. doi: 10.1123/ijspp.2015-0176

Sáez de Villarreal, E., Requena, B., \& Cronin, J. B. (2012). The Effects of Plyometric Training on Sprint Performance: A Meta-Analysis. Journal of Strength \& Conditioning Research, 26(2), 575-584. doi: 10.1519/ JSC.0b013e318220fd03

Thomas, K., French, D., \& Hayes, P. R. (2009). The effect of two plyometric training techniques on muscular power and agility in youth soccer players. Journal of strength and conditioning research, 23(1), 332-335. doi: 10.1519/JSC.0b013e318183a01a

van den Tillaar, R., Lerberg, E., \& von Heimburg, E. (in Press). Comparison of three types of warm-up upon sprint ability in experienced soccer players. Journal of Sport and Health Science. doi: 10.1016/j. jshs.2016.05.006

Wilson, G. J., Newton, R. U., Murphy, A. J., \& Humphries, B. J. (1993). The optimal training load for the development of dynamic athletic performance. Medicine and Science in Sports and Exercise, 25(11), 12791286.

Wong, P.-l., Chamari, K., \& Wisløff, U. (2010). Effects of 12-Week On-Field Combined Strength and Power Training on Physical Performance Among U-14 Young Soccer Players. Journal of Strength \& Conditioning Research, 24(3), 644-652. doi: 10.1519/JSC.0b013e3181ad3349 